\title{
Optimization of bioconversion process for trehalose production from enzymatic hydrolysis of kudzu root starch using a visualization method
}

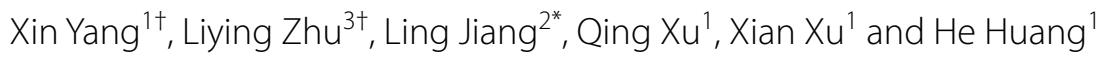

\begin{abstract}
Background: Trehalose has many advantages due to its inertness and the ability to stabilize biomolecules. Trehalose synthase can catalyze intramolecular rearrangement of the inexpensive maltose into trehalose in a single step, which represents a simple, fast, and low-cost method for the future industrial production of trehalose.

Results: In this work, an intelligent visualization method for producing trehalose via trehalose synthase was for the first time established and optimized by corresponding enzymatic hydrolysis with kudzu root starch as the initial raw material. For the first step, kudzu root starch was liquefied by $\alpha$-amylase at a certain dextrose equivalent value of $19-21$, and $\beta$-amylase and pullulanase to saccharify for a certain time, a four-factor nine-level experimental design with nine experiments was performed according to the uniform design table $\mathrm{U}_{9}^{*}\left(9^{4}\right)$ to optimize the yield of maltose. Then, optimization of trehalose conversion ratio from kudzu root starch hydrolysate was carried out with a four-factor 12-level experimental design to forecast the optimal process conditions. By comparing verification tests and predicted results, the optimized operating conditions were $\mathrm{pH} 7.1,22^{\circ} \mathrm{C}, 32.5 \%(14,000 \mathrm{U} / \mathrm{ml})$ loading amounts of Tres enzyme and after $24 \mathrm{~h}$, along with a $70.6 \%$ trehalose conversion rate.

Conclusions: The intelligent visualization method has succeeded in exploiting the conversion process of trehalose from kudzu root starch hydrolysate, which contributes significant benefits to the further commercial production of trehalose.
\end{abstract}

Keywords: Kudzu root starch, Enzymatic hydrolysis, Trehalose, Visualization method, Conversion rate

\section{Background}

Trehalose is a non-reducing disaccharide in which the two glucose unites are linked through an $\alpha, \alpha-(1,1)-$ glycosidic bond (Schiraldi et al. 2002). Production of this disaccharide is of commercial importance and opens a new field for its application in foods, cosmetics, and medicines, ranging from serving as a sweetener to a biomaterial stabilizer (Ohtake and Wang 2011). However, the conventional methods for the extraction from

\footnotetext{
*Correspondence: jiangling@njtech.edu.cn

${ }^{\dagger}$ Xin Yang and Liying Zhu contributed equally to this work

${ }^{2}$ College of Food Science and Light Industry, Nanjing Tech University, No.

30 Puzhunan Road, Nanjing 211816, China

Full list of author information is available at the end of the article
}

transformed plants and fermentation of yeast were not practical to produce trehalose on an industrial scale due to low yield and high cost. At the end of the last century, the Hayashibara Co. Ltd. has developed a two-step enzymatic system (trehalose-6-phosphate synthetase and trehalose-6-phosphate phosphatase) from a bacterial strain belonging to the genus Arthrobacter sp. Q36 isolated from soil sample (Maruta et al. 1995). The novel approach of trehalose production has led to a major reduction in the commercial price of trehalose from several hundred US\$ $/ \mathrm{kg}$ to 5-6 US\$ $/ \mathrm{kg}$, and for the first time, was successfully exploited in industrial production of trehalose. Nevertheless, further decrease in the production cost 
could be achieved by the application of other brand-new enzymatic route (Chang et al. 2012).

Except for the above two-enzyme system, four other main enzymatic routes are involved in trehalose biosynthesis: (1) maltooligosyltrehalose trehalohydrolase and maltooligosyltrehalose synthase (Fang et al. 2004); (2) trehalose phosphorylase (Carpinelli et al. 2006); (3) trehalose glycosyltransferring synthase (TreT) (Kouril et al. 2008), and (4) trehalose synthase (TreS) (Elbein et al. 2003). Among these routes, the intramolecular rearrangement of maltose into trehalose by TreS is a simple, fast, and low-cost method for the future industrial production of trehalose (Fig. 1). In our previous study, a novel TreS that comes from Deinococcus genus with high activity and conversion efficiency was identified and subsequently expressed in Escherichia coli (Jiang et al. 2013). Unfortunately, commercial utilization of trehalose is still hindered due to its high production cost compared with the other two disaccharides (Jiang et al. 2015). Since the carbon source employed in the culture medium has high share in the substrate cost, the exploitation of cheaply renewable agricultural residues has been strongly stimulated. Kudzu root is a rapid-growing and high-climbing perennial leguminous vine which is native to Eastern Asia (mainly in China, Japan, and Korea), and used as a forage crop and harvested for its root starch, fiber and medicinal qualities (Wang and Chen 2011). In general, fresh kudzu root contains $15-35 \%$ starch, which could be hydrolyzed by enzymes or acids to generate starch sugar (such as glucose, fructose, maltose syrups, cyclodextrins, and amylose) and starch derivatives (such as carboxy alkyl starch, ammonia alkyl starch, hydroxyalkyl starch and acetyl starch) (Allen 2003).

In recent years, more and more newly developed optimization strategies have been used to determine the optimal level of each experimental parameter. Compared with other statistical methods, uniform design (UD) reduces the number of experiments in a multiple-dimension optimization and allows the largest possible number of levels for each factor (Yan and Bogle 2007). The intelligent visualization method is different from others in that the contours of the objective function can be viewed at the same time as the new mapping of the data from multi-dimensional space to a two-dimensional plane. The optimized operating point or region can be found intuitively according to the distribution of the objective function. Then the near-optimal point can be mapped back to the original multi-dimensional space with an inversion mapping method, and will be represented in terms of original variables (Shi et al. 2009). The visualization method was applied to solve two types of problems, one is the determination of optimal operation point or region for a chemical process and the other is optimization of multi-objective problems (Zhang et al. 2010). In this study, kudzu root starch was treated by a series of enzymatic hydrolysis to produce maltose, which was further transformed into trehalose by TreS. With the data obtained from single-factor experiments and uniform design, we fully took the advantages of the intelligent visualization software to fit the data and obtained the optimal yield point of trehalose production.

\section{Methods}

\section{Materials}

Kudzu root starch was supplied from Hunan Province, China, in November 2014. Enzymes of $\alpha$-amylase (EC 3.2.1.1, 1 unit to liberate $1.0 \mathrm{mg}$ maltose from starch in 3 min at $\mathrm{pH} 6.5$ at $75{ }^{\circ} \mathrm{C}$ in phosphate buffer) for liquefaction, $\beta$-amylase (EC 3.2.1.2, 1 unit to liberate $1.0 \mathrm{mg}$ maltose from starch in $3 \mathrm{~min}$ at $\mathrm{pH} 4.8$ at $60{ }^{\circ} \mathrm{C}$ in acetate buffer) and pullulanase (EC 3.2.1.41, 1 unit to liberate $1.0 \mathrm{mmol}$ of maltotriose from pullulan/min at $\mathrm{pH} 4.8$ at $60{ }^{\circ} \mathrm{C}$ in acetate buffer) for saccharification were purchased from Aladdin Industrial Co. (Shanghai, China). The recombinant E. coli BL21 (DE3) harboring pET22b(+)-treS, in which the treS gene encoding the TreS from Deinococcus desert, was constructed previously in our laboratory (Jiang et al. 2013).

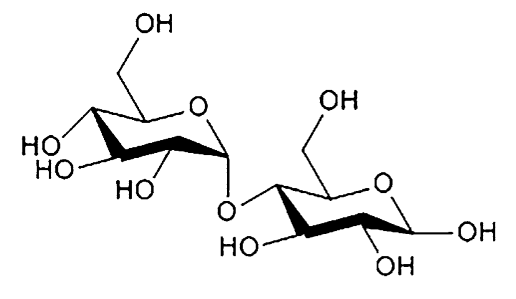

maltose
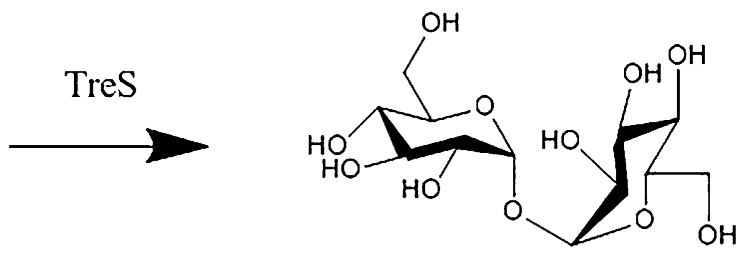

trehalose

Fig. 1 Overview of trehalose synthesis from maltose by trehalose synthase. 


\section{Preparation of the hydrolysate of kudzu root starch}

Twenty gram kudzu root starch was mixed with $100 \mathrm{ml}$ deionized water to total weight of $120 \mathrm{~g}$. Adjusting the slurry to $\mathrm{pH} 6.9$ by $1 \mathrm{~mol} / \mathrm{l} \mathrm{HCl}$ or $1 \mathrm{~mol} / \mathrm{l} \mathrm{NaOH}$. Heating to $75^{\circ} \mathrm{C}$ until kudzu root starch was dissolved completely, then $\alpha$-amylase was added for liquefaction. The reaction condition parameters of the liquefaction were $150 \mathrm{U} \alpha$-amylase/g starch at $75^{\circ} \mathrm{C}$, and pH 6.5 (Soni et al. 2003). After cooling to $60{ }^{\circ} \mathrm{C}, \mathrm{pH}$ of the mixture was adjusted to 4.8 with $1 \mathrm{~mol} / \mathrm{l} \mathrm{HCl}$. Then, different amounts of $\beta$-amylase and pullulanase were added for saccharification, the resulting syrup was maintained at $60{ }^{\circ} \mathrm{C}$ and was continually stirred using a magnetic stirrer and aliquots were removed at time intervals of $2,4,6,8,10,12,14$ and $15 \mathrm{~min}$ for dextrose equivalent (DE) analysis (Lin et al. 2011).

\section{Visualization method}

Kudzu root starch was used as the substrate of conversion of maltose. There are four important factors influencing the value of conversion rate, namely $\mathrm{DE}\left(X_{1}\right)$, the amount of $\beta$-amylase $\left(X_{2}\right)$ and pullulanase $\left(X_{3}\right)$, as well as the saccharification time $\left(X_{4}\right)$. At first, a four-factor ninelevel experimental design with nine experiments was performed according to the UD table $U_{9}^{\prime \prime}\left(9^{4}\right)$. The experimental design and results as well as the predicted outputs were calculated by the intelligent visualization method (Version 1.0, China) (Jiang et al. 2015). Similarly, kudzu root starch hydrolysate was used as the catalytic substrate of trehalose production. There are four important factors influencing the value of conversion rate, namely $\mathrm{pH}\left(X_{1}\right)$, reaction temperature $\left(X_{2}\right)$, enzyme concentration $\left(X_{3}\right)$, and reaction time $\left(X_{4}\right)$. A four-factor 12-level experimental design with 12 experiments was performed according to the UD table $\mathrm{U}_{12}^{*}\left(12^{4}\right)$.

The basic principles of visualization method are shown in Additional file 1: Figure S1. Firstly, the simple data in multi-dimensional space are mapped to a twodimensional plane with a mapping model, in which the contours of the objective functions are generated automatically. According to the distribution of the contours, the optimized operating direction or region can then be located intuitively. Finally, an optimal point found in the above region can be mapped back to the original multidimensional space with an inversion mapping method, which will be represented in terms of original variables.

The above mapping process is established based on a neural network as shown in Fig. 2. For an optimization problem with an m-dimensional input vector, $\mathbf{x}$, and an 1-dimensional output vector, $\mathbf{y}$, the data are mapped to two new variables, $z_{1}$ and $z_{2}$, in a two-dimensional mapping plane as follows:

$$
z_{1}=\omega_{1} X^{T}, z_{2}=\omega_{2} X^{T}
$$

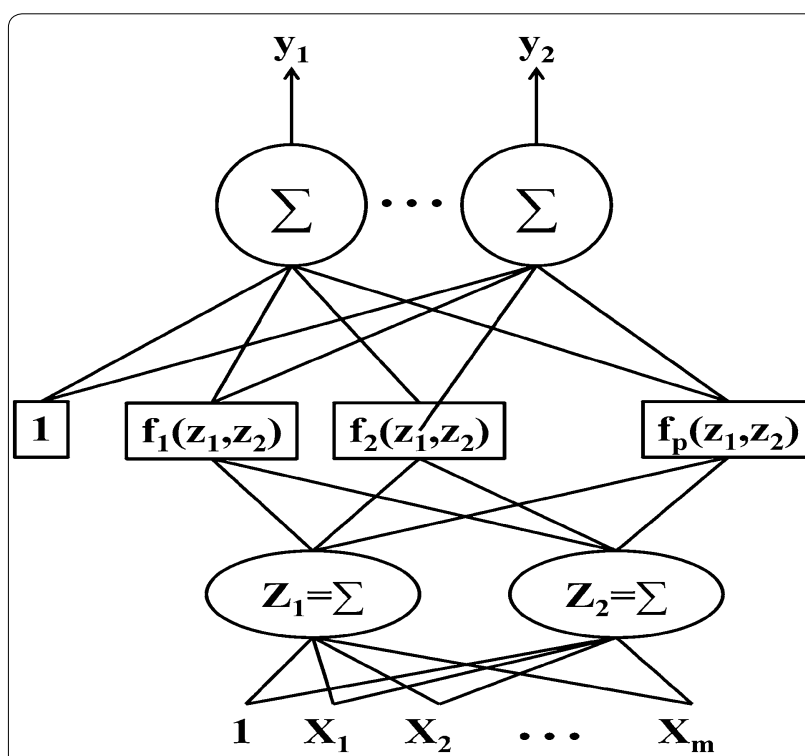

Fig. 2 Mapping model.

$$
Y=V P^{T}=\left[\begin{array}{cccc}
v 10 & v 11 & \cdots & v 1 p \\
v 20 & v 21 & \cdots & v 2 p \\
\cdots & \cdots & \cdots & \cdots \\
v l 0 & v l 1 & \cdots & v l p
\end{array}\right]\left[\begin{array}{c}
1 \\
f 1(z 1, z 2) \\
\cdots \\
f p(z 1, z 2)
\end{array}\right]
$$

where $\quad X=\left[1, x_{1}, x_{2}, \ldots, x_{m}\right], w_{j}=\left[w_{0 j}, w_{1 j}, w_{2 j}, \ldots\right.$, $\left.w_{m j}\right], j=1,2, Y=\left[y_{1}, y_{2}, \ldots, y_{l}\right], P=\left[1, f_{1}\left(z_{1}, z_{2}\right), f_{2}\left(z_{1}\right.\right.$, $\left.\left.z_{2}\right), \ldots, f_{p}\left(z_{1}, z_{2}\right)\right], V=\left[v_{1}, v_{2}, \ldots, v L\right]_{L \times p}^{T}, v_{k}=\left[v_{k 0}, v_{k 1}\right.$, $\left.\ldots, v_{k(p+1)}\right], k=1,2, \ldots, l$, where $X$ is an $\mathrm{m}$-dimensional input vector, $Y$ is an l-dimensional output vector, $z_{1}$ and $z_{2}$ are two variables on mapping plane, $w_{1}, w_{2}$ and $V$ are the weight vectors of the neural network, $P$ is a nonlinear extending vector for enhancing the mapping effect. First, m-dimensional input vector $X$ is first mapped to a two-dimensional plane described with vector $z\left(=\left[z_{1}, z_{2}\right]\right)$. Then $z$ is mapped to $\mathrm{n}$-dimensional output $Y$ through nonlinear mapping. In this work, we take simply $P=\left[1, f_{1}\left(z_{1}, z_{2}\right), f_{2}\left(z_{1}, z_{2}\right), \ldots, f_{p}\left(z_{1}, z_{2}\right)\right]=\left[1, z_{1}, z_{2}\right.$, $\left.z_{1}^{2}, z_{2}^{2}, z_{1} z_{2}\right]$.

Therefore, it is able to use the two variables $z_{1}$ and $z_{2}$ to describe the contour of the objective function $Y$ on the plane in case of the neural network trained with sample data. According to the contour distribution of the objective function on the plane, it is easy to decide the optimal point or region on the plane. Based on the linear relationship between $x$ and $z$, the optimal point $c$ on the plane can be mapped inversely to the original multi-dimensional space by the following equation:

$$
x^{c}=x^{a}+\beta\left(x^{b}-x^{a}\right)
$$


where $x^{a}, x^{b}$ and $x^{c}$ are points in multi-dimensional space corresponding to points $a, b$ and $c$. $\beta$ is the step size, which equals to the ratio of the distance between points $a$ and $c$ to that between points $a$ and $b$ :

$$
\beta=\frac{\overline{a c}}{\overline{a b}}
$$

where $0 \leq \beta \leq 1$ for interpolation and $\beta \geq 1$ for extrapolation. In practical applications, first two reference points on the plane are selected in the optimization direction, and then the step size is determined to reach the optimal point. Given an optimization direction and step size, one can calculate the corresponding optimal point in the original multi-dimensional space.

\section{Enzyme assay}

The recombinant TreS was constructed in Escherichia coli BL21(DE3), which was described in detail in our previous study (Jiang et al. 2013). The E. coli cells were harvested by centrifugation at $12,000 \times g$ for $20 \mathrm{~min}$ at $4{ }^{\circ} \mathrm{C}$ and suspended in PBS buffer $\left(40 \mathrm{mM} \mathrm{NaH}_{2} \mathrm{PO}_{4}{ }^{-}\right.$ $\mathrm{Na}_{2} \mathrm{HPO}_{4}, \mathrm{pH}$ 7.0) followed by sonification and centrifugation to remove insoluble cell debris. The supernatant was filtered through a $0.45-\mu \mathrm{m}$ filter and the recombinant TreS was purified through a Ni-NTA affinity chromatography column according to the manufacturer's purification protocol manual (Novagen, Ni-NTA His•Bind Resins). The purified TreS was resuspended in PBS buffer with the fixed the enzyme activity of $14,000 \mathrm{U} / \mathrm{ml}$. The standard reaction was performed by adding $1.5 \mu \mathrm{l}$ of purified TreS into $50 \mu \mathrm{l}$ of reaction solution containing in $50 \mathrm{mM}$ sodium phosphate buffer with different $\mathrm{pH}$ values and $150 \mathrm{mM}$ maltose and incubating at different temperatures. The reaction was terminated by heating the mixture in boiling water for $15 \mathrm{~min}$. One unit (U) of enzyme activity was defined as the amount of enzyme that catalyzes the formation of $1 \mathrm{nmol}$ trehalose per min in $1 \mathrm{ml}$ system under the specified conditions. The trehalose conversion rate (CR) was calculated by the ratio of the trehalose product to the amount of maltose substrate.

\section{Analytical methods}

The starch solubility and the content of maltose in the hydrolysate were determined by DE, which was analyzed using the Lane-Eynon method (Yildiz et al. 2005). The maltose yield rate (YR) was calculated by the ratio of the maltose product to the amount of starch substrate. The amount of trehalose, glucose, and maltose was measured using a HPLC (Dionex) system equipped with a Sepax HP-Amino column and a refractive index detector. The mobile phase was acetonitrile-water (75:25). The flow rate was $0.6 \mathrm{ml} / \mathrm{min}$ and column temperature was $30{ }^{\circ} \mathrm{C}$.
The resulting data were analyzed off-line with Origin 6.0 software (Microcal, Northampton, MA, USA). All experiments were carried out in duplicate or triplicate.

\section{Results and discussion Kudzu root starch hydrolysis}

With respect to starch content and yield, kudzu is considered to rival the carbohydrate production from maize and sugar cane (Sage et al. 2009). For a better trehalose production, a higher maltose concentration with a lower glucose concentration was preferable with kudzu starch hydrolysate as the substrate. Previous studies suggested that the liquefaction DE value of starch was found to influence the saccharide composition, and the optimum liquefaction DE of starch for maltose syrup production was 8-11 (Marchal et al. 1999). Both a too high and too low DE value can significantly affect the production of maltose, the former one causes a rapid decrease of maltose yield, while the latter one results in a high viscosity which is harmful to the following saccharification process (Besselink et al. 2008). In this study, $\alpha$-amylase was used to hydrolyze kudzu root starch granules to obtain utmost maltose. Our preliminary experiments showed that it needed only 8 min of liquefaction time to reach a $\mathrm{DE}$ value of 8-11 with kudzu root starch, which was faster than rice and corn starch (e.g., 22-27 min) (Additional file 2: Figure S2) (Roy and Gupta 2004). Small granules in kudzu root could be the most possibly reason to increase enzyme susceptibility and easily to be liquefied (Kim and Robyt 1999). However, the optimal DE value was finally selected among 19-21 to obtain the utmost starch utilization and maltose content in the present study (Additional file 3: Figure S3, Additional file 4: Table S1).

After liquefaction of kudzu root starch, different amounts of $\beta$-amylase and pullulanase were added for saccharification. As we all know, $\beta$-amylase, debranching enzyme, can hydrolyze $\alpha-1,4$ links dextrins and pullulanase can specifically cleave certain $\alpha-1,6$ links in starch for more maltose production. Various combinations of $\mathrm{DE}$ value, $\beta$-amylase and pullulanase amount, as well as the saccharification time are listed in Table 1 according to the orthogonal design scheme, together with the experimental results. Figure 3 shows the mapping diagram of hydrolysis yield of kudzu root starch. Sample data in multi-dimensional space were mapped and reduced to a two-dimensional plane by the visualization method, with nine blue points (hollow points) representing nine groups of experiments, respectively. The lines formed an elliptical shape with the increment directed from both the top left corner and the bottom right corner to the center. The optimal result (e.g., point 2 and 3) can be intuitively observed in the mapping diagram, and the optimal reaction conditions could be obtained by 
Table 1 Uniform design scheme and results of maltose yield rate

\begin{tabular}{|c|c|c|c|c|c|}
\hline Sample & $x_{1}$ & $\begin{array}{l}X_{2} \\
\text { (U/g starch) }\end{array}$ & $\begin{array}{l}x_{3} \\
\text { (U/g starch) }\end{array}$ & $X_{4}(\mathrm{~h})$ & $\begin{array}{l}\text { Factors } \\
\text { index } \\
\text { YR (\%) }\end{array}$ \\
\hline 1 & 19 & 25 & 0.5 & 24 & 76 \\
\hline 2 & 19 & 30 & 1 & 36 & 92 \\
\hline 3 & 19 & 35 & 1.5 & 48 & 92 \\
\hline 4 & 20 & 25 & 1 & 48 & 88 \\
\hline 5 & 20 & 30 & 1.5 & 24 & 85 \\
\hline 6 & 20 & 35 & 0.5 & 36 & 86 \\
\hline 7 & 21 & 25 & 1.5 & 36 & 85 \\
\hline 8 & 21 & 30 & 0.5 & 48 & 88 \\
\hline 9 & 21 & 35 & 1 & 24 & 85 \\
\hline
\end{tabular}

$X_{1}$ is the value of $\mathrm{DE}, X_{2}$ is the amount of $\beta$-amylase, $X_{3}$ is the amount of pullulanase, and $X_{4}$ is saccharification time.

$Y R$ maltose yield rate.

inversely mapping the optimal point in the two-dimensional plane to original multi-dimensional space. Value of the optimal predicted point by extrapolating from point 1 to 3 with step size 0.5 was $93.8 \%$ of maltose yield with the hydrolytic conditions of 19 of DE, $32.5 \mathrm{U} / \mathrm{g}$ starch of $\beta$-amylase, $1.25 \mathrm{U} / \mathrm{g}$ starch of pullulanase, and $42 \mathrm{~h}$ of saccharification time (Table 2). The value of these factors at the predicted point is inside the operating bound which indicates that the predicted point is feasible. Also, the result from the verification experiment with the same
Table 2 Comparison of prediction results and test results of maltose yield rate

\begin{tabular}{|c|c|c|c|c|c|}
\hline Sample & $X_{1}$ & $X_{2}$ (U/g starch) & $X_{3}$ (U/g starch) & $X_{4}(\mathrm{~h})$ & YR (\%) \\
\hline Predicted data & 19 & 32.5 & 1.25 & 42 & 93.8 \\
\hline Real data & 19 & 32.5 & 1.25 & 42 & 94.6 \\
\hline
\end{tabular}

hydrolytic parameters came to the highest value of $94.6 \%$ in terms of maltose yield from kudzu root starch hydrolysates. Sugar compositions and the content of kudzu root starch hydrolysate are also shown in Table 3. The process of saccharification will inevitably produce a small quantity of glucose (about $3.5 \%$ ), which has been proved to inhibit the conversion efficiency of TreS (Wang et al. 2007).

\section{Optimize the process of trehalose production}

So far, a number of TreSs from several bacterial strains have been identified and characterized (Jiang et al. 2013). However, these TreSs are still not satisfying in a practical application with regard to conversion efficiency, because they catalyze the conversion of maltose into trehalose in a reversible reaction, together with a side reaction of hydrolysis to irreversibly form glucose. Therefore, in the process of trehalose production, the degree of trehalose conversion rate $(\mathrm{CR})$ was regarded as the most important

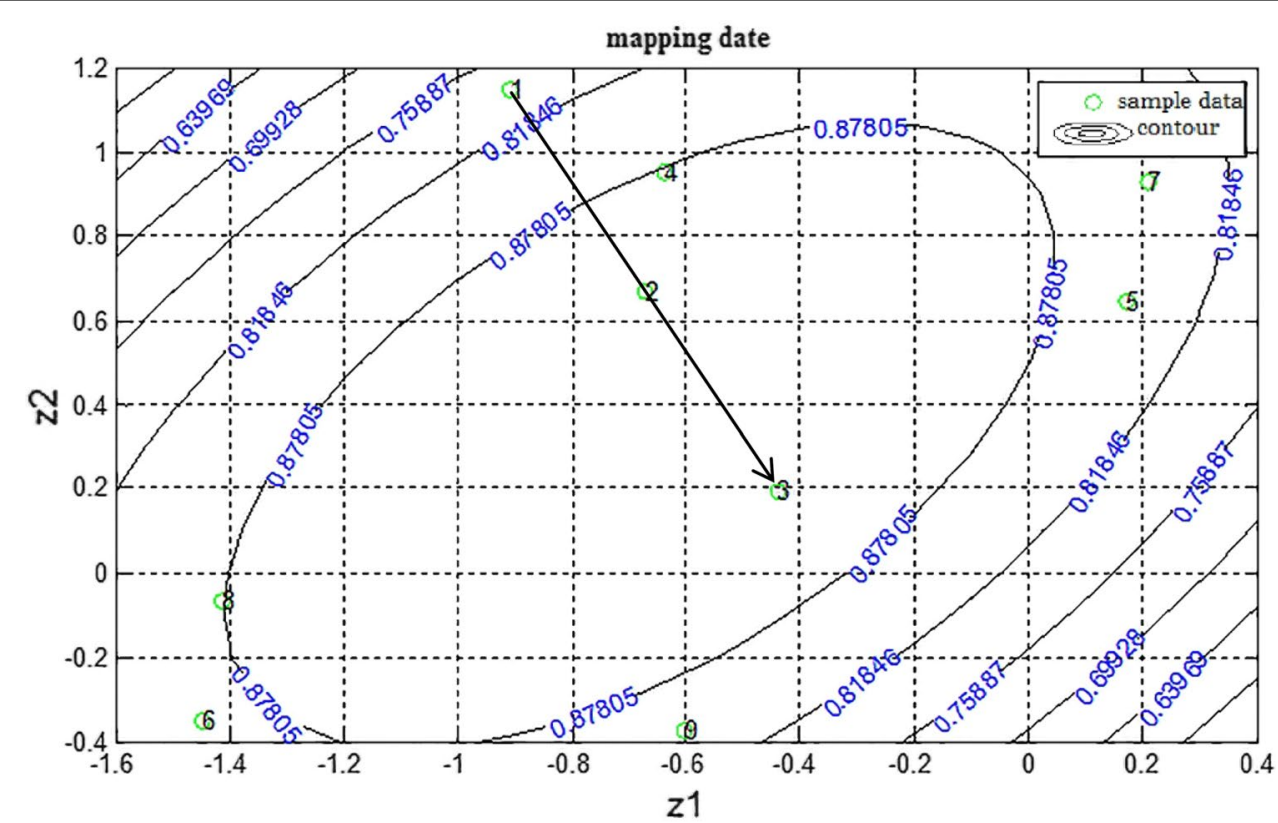

Fig. 3 Contours on the mapping plane for the yield rate of maltose. The full line represented contours, small circles and numbers represented sample data, arrows represented the direction of data optimizing. 
Table 3 Sugar contents of kudzu root starch hydrolysate

\begin{tabular}{lc}
\hline Starch hydrolysate & Sugar compositions (\%) \\
\hline Maltose & $94.6 \pm 2.5$ \\
Glucose & $3.5 \pm 0.04$ \\
$\begin{array}{l}\text { Other sugars (larger than } \\
\text { those of trisaccharide) }\end{array}$ & $1.9 \pm 0.03$ \\
\hline
\end{tabular}

Table 4 Uniform design scheme and results of trehalose conversion rate

\begin{tabular}{llllll}
\hline Sample & $\boldsymbol{X}_{\mathbf{1}}$ & $\boldsymbol{X}_{\mathbf{2}}\left({ }^{\circ} \mathbf{C}\right)$ & $\boldsymbol{X}_{\mathbf{3}}(\mathbf{\%})$ & $\boldsymbol{X}_{\mathbf{4}}(\mathbf{h})$ & $\begin{array}{l}\text { Factors index } \\
\text { CR (\%) }\end{array}$ \\
\hline 1 & 6 & 25 & 30 & 36 & 57.6 \\
2 & 6 & 40 & 10 & 30 & 38.9 \\
3 & 6.5 & 25 & 40 & 24 & 65.7 \\
4 & 6.5 & 40 & 20 & 18 & 42.6 \\
5 & 7 & 20 & 10 & 36 & 52.7 \\
6 & 7 & 35 & 30 & 30 & 57.6 \\
7 & 7.5 & 20 & 20 & 24 & 62.3 \\
8 & 7.5 & 35 & 40 & 18 & 56.3 \\
9 & 8 & 15 & 30 & 36 & 47.2 \\
10 & 8 & 30 & 10 & 30 & 48.4 \\
11 & 8.5 & 15 & 40 & 24 & 33.7 \\
12 & 8.5 & 30 & 20 & 18 & 47.9
\end{tabular}

$X_{1}$ is $\mathrm{pH}, X_{2}$ is reaction temperature, $X_{3}$ is enzyme concentration, and $X_{4}$ is reaction time.

$C R$ trehalose conversion rate. property. The reaction condition parameters of the intramolecular transglycosylation from maltose into trehalose by TreS were summarized in many previous studies and listed in Table 4 (Jiang et al. 2013; Ma et al. 2006; Lee et al. 2005; Xiuli et al. 2009; Wang et al. 2007b, c; Zhu et al. 2008). Various combinations of $\mathrm{pH}(6-8.5)$, reaction temperature $\left(15-40{ }^{\circ} \mathrm{C}\right)$, TreS concentration $(10-40 \%$, $14,000 \mathrm{U} / \mathrm{ml})$ and reaction time $(18-36 \mathrm{~h})$ are shown in Table 4 according to the orthogonal design scheme, together with the experimental results.

Figure 4 presents the mapping diagram of trehalose yield with maltose as the substrate, which was obtained from kudzu root starch hydrolysate. The objective function as shown in Fig. 4 is a hyperbola, and the center is a saddle point. Twelve green points in figure represent 12 groups of trehalose production tests, respectively. It is clear that a high conversion rate can be obtained by decreasing $z_{1}$. Taking points 10 and 3 as references and step size as 1.6, a predicted point is obtained through extrapolation in the direction of the arrow. The predicted data and the corresponding reaction parameters are listed in Table 5. The results from the verification experiment indicated that the optimal conditions of trehalose production are $\mathrm{pH} 7.1$, temperature of $22{ }^{\circ} \mathrm{C}, 32.5 \%$ $(14,000 \mathrm{U} / \mathrm{ml})$ of TreS enzyme loading amounts and for $24 \mathrm{~h}$, trehalose conversion rate from kudzu root starch hydrolysate could reach up to $70.6 \%$, which was among the highest values ever produced in the enzymatic catalysis process (Jiang et al. 2013).

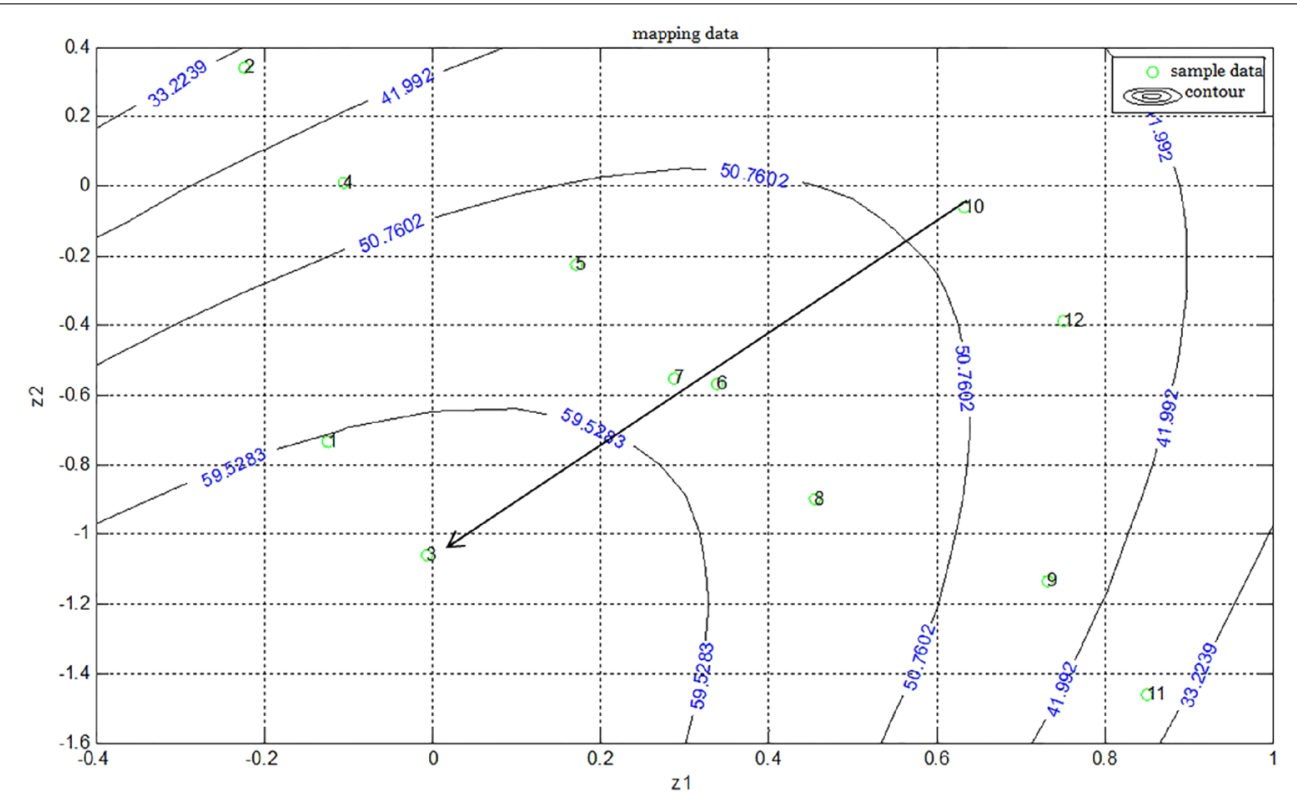

Fig. 4 Contours on the mapping plane for the conversion rate of trehalose. The full line represented of contours, small circles and numbers represented of sample data, arrows represented of the direction of data optimizing. 
Table 5 Comparison of prediction results and test results of trehalose conversion rate

\begin{tabular}{llllll}
\hline Sample & $\boldsymbol{X}_{\mathbf{1}}$ & $\boldsymbol{X}_{\mathbf{2}}\left({ }^{\circ} \mathbf{C}\right)$ & $\boldsymbol{X}_{\mathbf{3}}(\boldsymbol{\%})$ & $\boldsymbol{X}_{\mathbf{4}}(\mathbf{h})$ & $\mathbf{C R}(\%)$ \\
\hline Predicted data & 7.1 & 22 & 32.5 & 24 & 69.8 \\
Real data & 7.1 & 22 & 32.5 & 24 & 70.6
\end{tabular}

$X_{1}$ is $\mathrm{pH}, X_{2}$ is reaction temperature, $X_{3}$ is enzyme concentration, and $X_{4}$ is reaction time.

$C R$ trehalose conversion rate.

\section{Conclusion}

In conclusion, an integrated bio-process of trehalose production from kudzu root starch through two-stage enzymatic hydrolysis into malt syrup was established with the help of the intelligent visualization method in this study. The high-yield of $94.6 \%$ maltose was realized by $\alpha$-amylase, $\beta$-amylase and pullulanase pretreatment under the optimum DE of 19 and saccharificaiton condition of $32.5 \mathrm{U} \beta$-amylase/g starch and 1.25 $\mathrm{U}$ pullulanase/g starch. Furthermore, the optimum conditions of trehalose production were obtained with the maximum trehalose conversion rate of $70.6 \%$, which was considered as a good candidate for the large-scale production of trehalose in the near future. Thus, application of the visualization method for optimization of parameters of enzyme catalytic reaction conditions was proved successful, and its further application in biocatalysis technology was expected.

\section{Additional files}

Additional file 1: Figure S1. Principles of visualization method.

Additional file 2: Figure S2. Variation of dextrose equivalent with liquefaction time. By comparing the time of kudzu starch liquefaction in this work with that of rice and corn starch liquefaction obtained from previous studies, it could be concluded it was easier for kudzu root starch to be liquefied than rice and corn starch by enzymes (e.g., $\alpha$-amylase, $\beta$-amylase and pullulanase)

Additional file 3: Figure S3. Effects of DE values on the yield of maltose content after liquefaction and saccharification. With the increasing of DE value, the content of maltose was declined. Hence, for a high maltose yield, a low DE value should be selected. Overall considering the starch utilization and maltose content, the optimal DE value was selected at 19-21.

Additional file 4: Table S1. Effect of DE value on starch solubility of kudzu root starch.

\section{Authors' contributions}

$X Y$ carried out the experiments. $X Y$ and $L Y Z$ analyzed the data and wrote the manuscript. QX and XX carried out the drawing and have revised the manuscript. $\mathrm{LJ}$ and $\mathrm{HH}$ conceived the study and helped to draft the manuscript, and have revised the manuscript. All authors read and approved the final manuscript.

\section{Author details}

${ }^{1}$ College of Biotechnology and Pharmaceutical Engineering, Nanjing Tech University, No. 30 Puzhunan Road, Nanjing 211816, China. ${ }^{2}$ College of Food Science and Light Industry, Nanjing Tech University, No. 30 Puzhunan Road, Nanjing 211816, China. ${ }^{3}$ College of Sciences, Nanjing Tech University, No. 30 Puzhunan Road, Nanjing 211816, China.

\section{Acknowledgements}

We thank Prof. Liexiang Yan (School of Chemical Engineering, Wuhan University of Technology, 430070 Wuhan, China) for providing the software of visualization method. The project was supported by the National Science Foundation for Distinguished Young Scholars of China (21225626), the Natural Science Foundation of Jiangsu Province (BK20131406, BK20130917), the Natural Science Foundation for Colleges and Universities in Jiangsu Province (14KJB530003), the Ministry of Education Research Foundation for the Doctoral Program (20123221120011).

\section{Compliance with ethical guidelines}

\section{Competing interests}

The authors declare that they have no competing interests.

Received: 14 May 2015 Accepted: 3 August 2015

Published online: 18 August 2015

\section{References}

Allen SJ (2003) Comparison of optimised isotherm models for basic dye adsorption by kudzu. Bioresour Technol 88:143-152

Besselink T, Baks T, Janssen AE, Boom RM (2008) A stochastic model for predicting dextrose equivalent and saccharide composition during hydrolysis of starch by alpha-amylase. Biotechnol Bioeng 100:684-697

Carpinelli J, Krämer R, Agosin E (2006) Metabolic engineering of Corynebacterium glutamicum for trehalose overproduction: role of the TreYZ trehalose biosynthetic pathway. Appl Environ Microb 72:1949-1955

Chang SW, Liu PT, Hsu LC, Chen CS, Shaw JF (2012) Integrated biocatalytic process for trehalose production and separation from rice hydrolysate using a bioreactor system. Food Chem 134:1745-1753

Elbein AD, Pan YT, Pastuszak I, Carroll D (2003) New insights on trehalose: a multifunctional molecule. Glycobiology 13:17R-27R

Fang TY, Hung XG, Shih TY, Tseng WC (2004) Characterization of the trehalosyl dextrin-forming enzyme from the thermophilic archaeon Sulfolobus solfataricus ATCC 35092. Extremophiles 8:335-343

Jiang L, Lin M, Zhang Y, Li YP, Xu X, Li S (2013) Identification and characterization of a novel trehalose synthase gene derived from saline-alkali soil metagenomes. PLoS One 8:1-11

Jiang L, Cui HY, Zhu LY, Hu Y, Xu X, Li S et al (2015) Enhanced propionic acid production from whey lactose with immobilized Propionibacterium acidipropionici and role of trehalose synthesis in acid tolerance. Green Chem 17:250-259

Kim YK, Robyt JF (1999) Enzyme modification of starch granules: in situ reaction of glucoamylase to give complete retention of D-glucose inside the granule. Carbohydr Res 318:129-134

Kouril T, Zaparty M, Marrero J, Brinkmann H, Siebers B (2008) A novel trehalose synthesizing pathway in the hyperthermophilic Crenarchaeon Thermoproteus tenax: the unidirectional TreT pathway. Arch Microbiol 190:355-369

Lee JH, Lee KH, Kim CG, Lee SY, Kim GJ, Park YH et al (2005) Cloning and expression of a trehalose synthase from Pseudomonas stutzeri CJ38 in Escherichia coli for the production of trehalose. Appl Microbiol Biotechnol 68(2):213-219

Lin Q, Xiao H, Liu GQ, Liu Z, Li L, Yu F (2011) Production of maltose syrup by enzymatic conversion of rice starch. Food Bioprocess Tech 6:242-248

Ma Y, Xue L, Sun DW (2006) Characteristics of trehalose synthase from permeabilized Pseudomonas putida cells and its application in converting maltose into trehalose. J Food Eng 77(2):342-347 
Marchal LM, Van De Laar AMJ, Goetheer E, Schimmelpennink EB, Bergsma J, Beeftink HH et al (1999) Effect of temperature on the saccharide composition obtained after $\alpha$-amylolysis of starch. Biotechnol Bioeng 63:344-355

Maruta K, Nakada T, Kubota M, Chaen H, Sugimoto T, Kurimoto M et al (1995) Formation of trehalose from maltooligosaccharides by a novel enzymatic system. Biosci Biotechnol Biochem 59:1829-1834

Ohtake S, Wang YJ (2011) Trehalose: current use and future applications. J Pharm Sci 100:2020-2053

Roy I, Gupta MN (2004) Hydrolysis of starch by a mixture of glucoamylase and pullulanase entrapped individually in calcium alginate beads. Enzyme Microb Tech 34:26-32

Sage RF, Coiner HA, Way DA, Runion GB, Prior SA, Torbert HA (2009) Kudzu [Pueraria montana (Lour.) Merr. Variety lobata]: a new source of carbohydrate for bioethanol production. Biomass Bioenerg 33:57-61

Schiraldi C, Lernia I, Rosa M (2002) Trehalose production: exploiting novel approaches. Trends Biotechnol 20:420-425

Shi B, Yan L, Guo Q (2009) Application of visualization method to concrete mix optimization. Lect Notes Comput Sci 5553:41-48

Soni SK, Kaur A, Gupta JK (2003) A solid state fermentation based bacterial $\alpha$-amylase and fungal glucoamylase system and its suitability for the hydrolysis of wheat starch. Process Biochem 39(2):185-192

Wang L, Chen HZ (2011) Acetone-butanol-ethanol fermentation and isoflavone extraction using kudzu roots. Biotechnol Bioprocss E 16:739-745

Wang JH, Tsai MY, Chen JJ, Lee GC, Shaw JF (2007a) Role of the C-terminal domain of Thermus thermophilus trehalose synthase in the thermophilicity, thermostability, and efficient production of trehalose. J Agr Food Chem 55:3435-3443
Wang JH, Tsai MY, Lee GC, Shaw JF (2007b) Construction of a recombinant thermostable $\beta$-amylase-trehalose synthase bifunctional enzyme for facilitating the conversion of starch to trehalose. J Agr Food Chem 55(4):1256-1263

Wang JH, Tsai MY, Chen JJ, Shaw JF (2007c) Role of the C-terminal domain of Thermus thermophilus trehalose synthase in the thermophilicity, thermostability, and efficient production of trehalose. J Agr Food Chem 55(9):3435-3443

Xiuli W, Hongbiao D, Ming Y, Yu Q (2009) Gene cloning, expression, and characterization of a novel trehalose synthase from Arthrobacter aurescens. Appl Microbiol Biotechnol 83(3):477-482

Yan LX, Bogle D (2007) A visualization method for operating optimization. Comput Chem Eng 31:808-814

Yildiz HB, Kiralp S, Toppare L, Yagci Y (2005) Immobilization of invertase in conducting polypyrole/PMMA-co-PMTM graft copolymers. J Appl Polym Sci 96:502-507

Zhang G, She Y, You Y, Yan L, Shi B (2010) The application of an advanced visualized method in synthesis process optimization of carboxymethyl hydroxyethyl starch. Carbohydr Polym 79:673-676

Zhu Y, Zhang J, Wei D, Wang Y, Chen X, Xing L et al (2008) Isolation and identification of a thermophilic strain producing trehalose synthase from geothermal water in China. Biosci Biotechnol Biochem 72(8):2019-2024

\section{Submit your manuscript to a SpringerOpen ${ }^{\odot}$ journal and benefit from:}

- Convenient online submission

- Rigorous peer review

- Immediate publication on acceptance

- Open access: articles freely available online

- High visibility within the field

- Retaining the copyright to your article

Submit your next manuscript at $\boldsymbol{s p r i n g e r o p e n . c o m ~}$ 